\title{
A CONSTRUÇÃO DE UMA AGENDA DE GESTÃO COMPARTILHADA PARA A REOORGANIZAÇÃO DA DEMANDA EM SAÚDE BUCAL
}

\section{The construction of a shared management schedule to reorganise the oral health demand}

Lúcia Maria Lima Lemos de Melo - Mestre em Odontologia Preventiva e Social da Universidade Estadual Paulista "Júlio de Mesquita Filho", Faculdade de Odontologia de Araçatuba, SP, Brasil. Especialista em Gestão; Especialista em Saúde da Família; Articuladora de Saúde Bucal do DRS II Araçatuba, Secretaria Estadual de Saúde, São Paulo, Brasil; E-mail: lumalim1@terra.com.br

Suzely Adas Saliba Moimaz • Professora Titular do Programa de Pós-Graduação em Odontologia Preventiva e Social da Universidade Estadual Paulista "Júlio de Mesquita Filho", Faculdade de Odontologia de Araçatuba, SP, Brasil. E-mail: sasaliba@foa.unesp.br

Cléa Adas Saliba Garbin • Coordenadora e Professora Titular do Programa de Pós-Graduação em Odontologia Preventiva e Social da Universidade Estadual Paulista "Júlio de Mesquita Filho", Faculdade de Odontologia de Araçatuba, Araçatuba, SP, Brasil. E-mail: cgarbin@foa.unesp.br

Artênio José Ísper Garbin - Professor Adjunto do Programa de Pós-Graduação em Odontologia Preventiva e Social da Universidade Estadual Paulista "Júlio de Mesquita Filho", Faculdade de Odontologia de Araçatuba, SP, Brasil. E-mail: agarbin@foa.unesp.br

Nemre Adas Saliba • Professora Titular do Programa de Pós-Graduação em Odontologia Preventiva e Social da Universidade Estadual Paulista "Júlio de Mesquita Filho", Faculdade de Odontologia de Araçatuba, SP, Brasil. E-mail: sasaliba@foa.unesp.br

Autora responsável pela correspondência:

Lúcia Maria Lima Lemos de Melo - lumalim1@terra.com.br

Revista Ciência Plural. 2016;2(1):42-55 


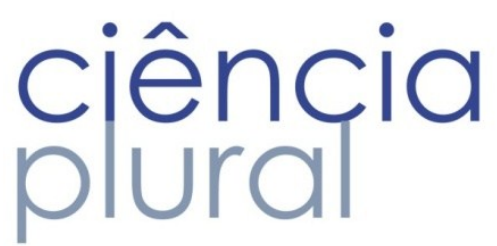

RESUMO

Introdução: A saúde bucal na Estratégia Saúde da Família representa um grande desafio para os gestores quando assumem o compromisso de desenvolver ações de promoção da saúde, prevenção de doenças e reabilitação dos indivíduos com a racionalização no uso dos recursos. A utilização de ferramentas de gestão faz-se necessária para garantir a equidade e integralidade das ações. Objetivo: 0 objetivo neste estudo foi avaliar a efetividade da implantação de uma agenda de atividades na melhoria do acesso aos serviços de saúde bucal. Métodos: Trata-se de uma pesquisa-ação realizada em um município do Estado de São Paulo, o qual foi selecionado com base nos seguintes critérios: existência de equipes de saúde bucal na Estratégia Saúde da Família, atenção primária e secundária em saúde bucal. Os dados foram obtidos por meio de análise do Plano Municipal de Saúde, do relatório do Programa de Monitoramento e Avaliação da Qualidade do Ministério da Saúde, entrevistas com o gestor, equipes de saúde e avaliação da produção ambulatorial. Resultados: Os resultados estão apresentados em três dimensões: construção da agenda; atividades realizadas e avaliação da agenda implantada. Foi realizada oficina para apresentação dos resultados e quatro meses antes e quatro meses após a implantação da agenda, foram avaliadas as seguintes variáveis em uma equipe de saúde bucal: primeira consulta programática, consultas de urgência, tratamentos completados e procedimentos realizados. Verificou-se que houve aumento de $63 \%$ na cobertura dos pacientes atendidos na primeira consulta, redução de $30 \%$ dos casos de urgência; aumento de $11 \%$ na cobertura de tratamentos completados e aumento no número de procedimentos. Conclusões: Concluiu-se que a agenda construída de acordo com os princípios e diretrizes do SUS foi facilitadora na reorganização do processo de trabalho promovendo a melhoria do acesso aos serviços de saúde bucal.

Palavras-chave: Saúde Bucal. Agenda de Saúde. Avaliação em Saúde. Gestão em Saúde.

\section{ABSTRACT}

Introduction: Oral health in the Family Health Strategy is a major challenge for managers when they are committed to developing health promotion, disease prevention and rehabilitation of individuals with the rational use of resources. The use of management tools is necessary to ensure the fairness and integrity of the shares. Objectives: The aim of this study was to evaluate the effectiveness of implementation of a schedule of activities to improve access to oral health services. Methods: This is an action research carried out in a municipality of São Paulo, which was selected based on the following criteria: existence of oral health teams in the Family Health Strategy, primary and secondary care in oral health. Data were obtained through analysis of the Municipal Health Plan, the Monitoring and Evaluation Programme of the Ministry of Health Quality report, interviews with the manager, health teams and evaluation of outpatient services. Results: The results are presented in three dimensions: construction agenda; activities and evaluation of the implanted agenda. Workshop was held to present the results months before and four months after the implementation of the agenda, we were assessed the following variables in a team of oral health: first programmatic consultation, emergency consultations, completed treatments and procedures performed. It was found that there was $63 \%$ increase in the coverage of patients seen at the first visit, $30 \%$ of emergency cases; increase of $11 \%$ in the supplemented treatments coverage and increased number of procedures. Conclusions: It was concluded that the built-in agenda in accordance with the principles and guidelines of SUS was facilitating the reorganization of the work process promoting improved access to oral health services.

Keywords: Oral Health. Health Agenda. Health Evaluation. Health Management. 


\section{Introdução}

O Programa Saúde da Família, atualmente reconhecido como Estratégia de Saúde da Família (ESF), é o modelo prioritário para a organização da Atenção Básica $(A B)^{1}$, baseado na corresponsabilidade da comunidade, equipes de saúde e gestor. A delimitação e o conhecimento do território e da população adstrita, o trabalho em equipe e a intersetorialidade constituem eixos fundamentais dessa concepção. ${ }^{2}$

Diante da necessidade de ampliar a atenção em saúde bucal da população brasileira, o Ministério da Saúde inseriu a saúde bucal na ESF proporcionando um modelo de vigilância à saúde que enfatiza a promoção da saúde e a prevenção das doenças ${ }^{3}$. A saúde bucal na ESF representa a possibilidade de criar um espaço de práticas e relações a serem construídas para a reorientação do processo de trabalho. 0 cuidado em saúde bucal passa a exigir a conformação de uma equipe de trabalho que se relacione com usuários e que participe da gestão dos serviços. ${ }^{4}$

Um grande desafio para os gestores quando a gestão é realizada sem planejamento, ficando a atenção atrelada à demanda espontânea. Nesse contexto, a utilização de ferramentas para a reorganização do processo de trabalho faz-se necessária para garantir a equidade e a integralidade das ações. Uma dessas ferramentas é a agenda, um recurso-chave para otimizar o tempo, priorizar e programar a atenção com base na análise das necessidades de saúde bucal da população ${ }^{5}$. Pode promover a organização da rotina do trabalho, compartilhamento da responsabilidade pelos resultados e possibilitar o acesso ao serviço de saúde com equidade.

A agenda é uma ferramenta de planejamento das ações da equipe de saúde bucal, solicitada na avaliação externa do Programa de Monitoramento e Avaliação da Qualidade (PMAQ) 6 realizado pelo Ministério da Saúde em parceria com Instituições de Ensino e Pesquisa.

Considerando as diretrizes da Política Nacional de Saúde Bucal que têm como pressupostos a garantia de ações resolutivas individuais, coletivas, de promoção e de prevenção, este estudo avaliou a agenda de atividades construída de forma compartilhada para a reorganização do acesso aos serviços de saúde bucal.

\section{Metodologia}

Tratou-se de uma pesquisa-ação, quanti-qualitativa, descritiva, realizada em um município do Estado de São Paulo, Brasil, cuja coleta de dados foi feita por meio de observações, análise documental e entrevistas utilizando-se um roteiro semi-estruturado, composto por questões abertas e fechadas.

A escolha do local da pesquisa teve como critérios: existência no município de serviços de atenção primária prestados pelas equipes de saúde bucal implantadas nas Equipes da Saúde da Família, atenção secundária de saúde bucal: Laboratório Regional de Prótese Dentária (LRPD), Centro de Especialidades Odontológicas (CEO) e facilidade de acesso ao serviço odontológico. O município selecionado foi Pereira Barreto por contemplar os critérios e ainda ter conquistado em 2013, o sexto lugar na categoria Nacional do Prêmio Brasil Sorridente e primeiro lugar na categoria estadual de municípios com até 50 mil habitantes, pelos trabalhos realizados pela Gestão Municipal na área da saúde bucal.

Os profissionais foram entrevistados individualmente, no local de trabalho: Unidades de Saúde da Familia, por um único pesquisador, que realizou a transcrição dos dados. A amostra final totalizou 19 profissionais: 0 gestor, profissionais das 6 Equipes de Saúde Bucal (ESB): 6 cirurgiões -dentistas, 6 auxiliares de saúde bucal e 


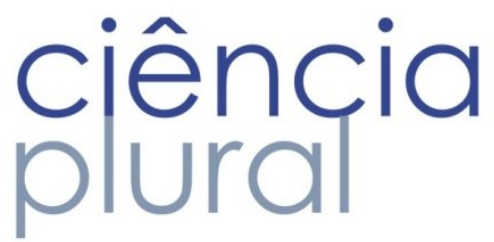

um agente comunitário de saúde de cada equipe da Saúde da Família com Saúde Bucal. Foi elaborada como instrumento de coleta de dados uma planilha no Microsoft Excell. Os dados quantitativos coletados foram apresentados em tabelas com valores numéricos e percentuais. Na análise qualitativa das questões abertas foi empregada a técnica de análise de conteúdo, descrita por Bardin?

A agenda de Saúde Bucal foi analisada nas respostas dos entrevistados, no Plano Municipal de Saúde Biênio 2014-2017 e no relatório do PMAQ-2013 (Programa de Monitoramento e Avaliação da Qualidade). Três dimensões foram definidas para a apresentação do resultado: construção da agenda, atividades realizadas e avaliação da agenda utilizada em uma ESB.

A agenda apresentada neste estudo (Quadro 1) foi efetivamente implantada no município em fevereiro de 2015. Na análise descritiva da agenda, os procedimentos odontológicos da atenção básica obtidos do relatório de produção ambulatorial municipal foram avaliados quatro meses antes e quatro meses após a implantação da agenda em uma Equipe de Saúde Bucal (ESB). Os critérios para a seleção da ESB foram: apresentação de dados completos da produção e não interrupção do atendimento odontológico por motivo de férias, licença ou outras atividades, no período analisado.

Por meio de oficina realizada in loco com os entrevistados, com carga horária de oito horas, os resultados foram apresentados e o tema da importância da agenda no planejamento e avaliação do processo de trabalho foi problematizado, tendo como fundamentos a literatura, as Diretrizes da Política Nacional da Atenção Básica ${ }^{1}$ e da Política Nacional de Saúde Bucal2.

O estudo foi aprovado pelo Comitê de Ética em Pesquisas com Seres Humanos, processo número 32482613.3.0000.5420, 2014 e os profissionais assinaram um Termo de Consentimento Livre e Esclarecido.

\section{Resultados}

\section{CONSTRUÇÃO DA AGENDA}

No questionário aplicado identificou-se nas respostas do gestor e dos cirurgiões-dentistas entrevistados o relato da dificuldade de implantação de uma agenda de atividades. Também foram identificadas a necessidade de implantação da agenda na análise documental do Plano Municipal de Saúde Biênio 2014-2017, no relatório do PMAQ-2013 (Programa de Monitoramento e Avaliação da Qualidade).

A primeira agenda utilizada no município foi elaborada por meio de oficinas pela equipe de saúde bucal em 2012-2013. Seguem trechos da fala do gestor que foram destacados para melhor compreensão dos problemas enfrentados no início da construção.

"Nessa agenda 2012-213 foram contemplados os grupos prioritários: criança, adolescente, gestante, idoso, hipertenso, diabético. Para os adultos que não se encaixavam nestes grupos não havia espaço na agenda, a não ser atendimento na urgência. O encaminhamento dos pacientes com hipertensão e diabete que apresentavam necessidade de atendimento odontológico era realizado pelos enfermeiros, porém pouco se encaminhava."

Esse fato provocou a reflexão sobre a necessidade de reorganização do agendamento. 


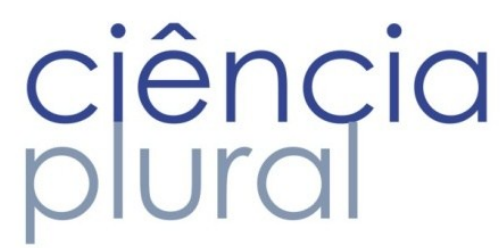

"Não houve discussão de critérios: seriam encaminhados todos os pacientes com hipertensão e diabetes? Por critério de risco? Todos os inscritos? Também não havia registro no prontuário de quem tinha sido encaminhado ou planilha de controle."

Assim, cada dentista foi adequando sua agenda de atividades sem uma discussão com a gestão para rever e adequar às necessidades do município.

O problema foi levado ao Conselho Municipal de Saúde, principalmente pelos representantes dos conselhos locais e houve questionamentos realizados pelos conselheiros:

"Por que quem precisa de atendimento não é atendido? Para ser atendido, precisa ser diabético e hipertenso?"

O Conselho de Saúde foi esclarecido sobre a organização da agenda de cada ESF, o problema de atendimento exclusivo a grupos prioritários foi discutido e houve a proposta de rever a agenda.

"Ao analisar os dados, percebeu-se que cada equipe estava trabalhando de uma maneira. Essa análise foi possivel porque os dentistas discutiram o problema de acordo com a realidade, com o que acontecia de fato na sua equipe."

A partir daí, a coordenação da saúde bucal se reuniu com as equipes e elaboraram uma nova proposta, que obteve resultados positivos conforme discurso do gestor:

"Atualmente, não há relato de reclamação na Secretaria de Saúde de usuários que não conseguem atendimento no dentista e esta era a grande reclamação no setor de saúde bucal."

A agenda apresentada no estudo foi construída e executada pela equipe de saúde bucal à partir do conhecimento do território de abrangência, do perfil populacional, diagnóstico das necessidades existentes, parâmetros e histórico da produção. Definiu-se o número de consultas de demanda espontânea e programada a serem disponibilizadas; o número de consultas destinadas aos retornos de rotina dos pacientes programados; as urgências; periodicidade das reuniões de equipe; as atividades administrativas; as atividades educativas e avaliação de risco das doenças bucais. Contemplava os grupos de gestantes, idosos, pacientes com diabetes, hipertensão, beneficiários do Programa Bolsa Família, escolares e bebês (Quadro 1). 


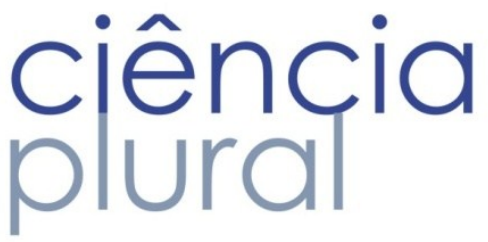

Quadro 1: Agenda de Saúde Bucal preconizada no município de Pereira Barreto, São Paulo.

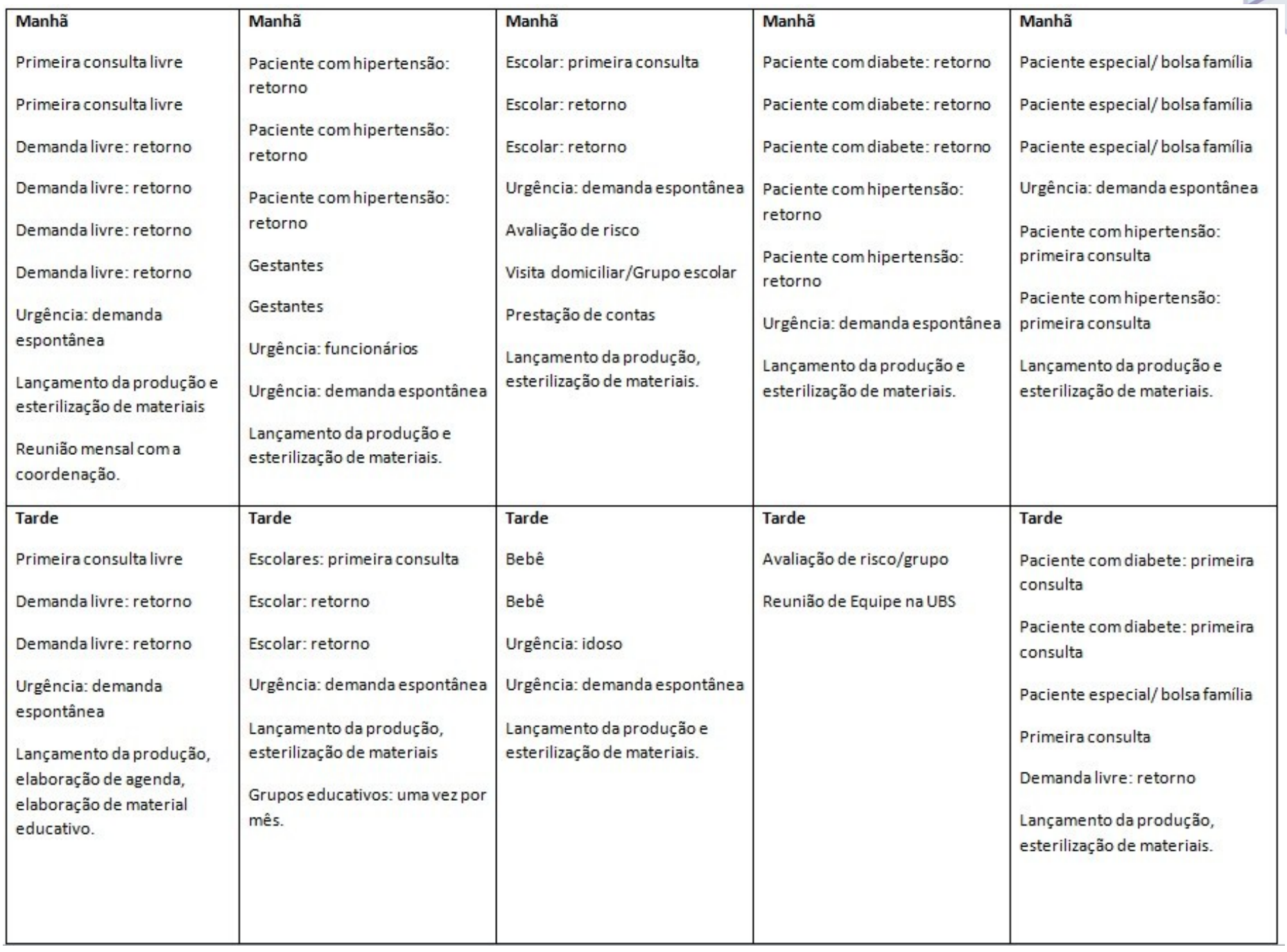

As seguintes modalidades de acesso dos pacientes às unidades de saúde foram identificadas na agenda implantada: urgências, escolares após triagem de risco realizada pelos cirurgiões-dentistas, agendamento de funcionários da própria unidade, agendamento pelo agente comunitário de saúde (ACS), livre demanda, retornos programados, grupos prioritários e contrarreferência (Quadro 2). 


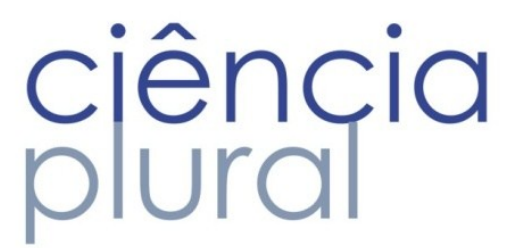

Quadro 2: Modalidades de acesso dos pacientes ao serviço odontológico nas Unidades de Saúde, Pereira Barreto, São Paulo.

\begin{tabular}{|l|l|}
\hline Modalidade & Descrição \\
\hline Urgência & Dor, edema na face, hemorragia, trauma dental, lesão bucal \\
\hline Escolares & $\begin{array}{l}\text { Avaliação de risco de cárie dentária, fluorose, doença } \\
\text { periodontal e de tecidos moles }\end{array}$ \\
\hline ACS & Busca ativa através do PSE, queixa \\
\hline Livre demanda & Procura imediata com posterior agendamento \\
\hline Retornos programados & Continuidade do tratamento \\
\hline Grupos prioritários & Avaliação de risco por indicação médica ou do enfermeiro \\
\hline Contrarreferência & Após atendimento no CEO \\
\hline Funcionários da UBS & Encaixe na agenda \\
\hline
\end{tabular}

\section{ATIVIDADES REALIZADAS}

A maioria dos cirurgiões-dentistas entrevistados relataram dificuldade em cumprir a agenda existente no município por causa da grande demanda. No entanto, a agenda foi reconhecida como um instrumento fundamental para a reorganização do processo de trabalho.

Outro fato relatado foi a dificuldade da população assimilar a odontologia como parte da equipe da saúde da família, ratificado na frase do ACS:

"A população não vê a Odontologia como parte da equipe da Saúde da Família."

Todos os cirurgiões-dentistas afirmaram que desenvolvem ações de saúde bucal para gestantes e idosos por meio de grupos prioritários da unidade básica de saúde (UBS). 0 atendimento a gestantes estava contemplado na agenda. A captação da gestante ocorria no momento que a mesma procurava a UBS para a realização do pré-natal, nos grupos prioritários e por encaminhamentos pelos ACS.

Quanto ao atendimento na atenção secundária, o paciente saía encaminhado da UBS com a consulta agendada no CEO e guia de referência e contrarreferência nas especialidades de endodontia e periodontia. Eram realizadas próteses parciais removíveis e totais por meio do Laboratório Regional de Prótese Dentária.

As metas mínimas de produção foram acordadas e pactuadas de forma compartilhada em reuniões de equipe com base em parâmetros e histórico de produção das equipes (Quadro 3). 


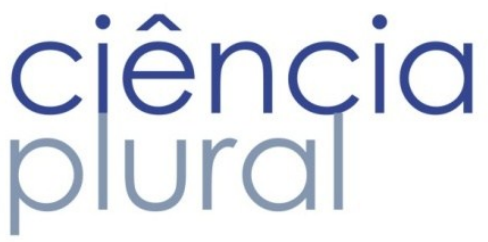

Quadro 3: Metas mínimas de produção em saúde bucal estabelecidas no município de Pereira Barreto, São Paulo.

\begin{tabular}{|l|c|}
\hline Atividades & Metas \\
\hline Procedimentos clínicos por hora & 2,38 \\
\hline Procedimentos ao dia & 19 \\
\hline Número de Primeiras Consultas Programadas ao mês & 25 \\
\hline Procedimentos ambulatoriais por mês & 380 \\
\hline Número de Tratamentos Concluídos ao mês & 20 \\
\hline Escovações ao mês pelos ACS & 50 \\
\hline Avaliações mensais de risco dos escolares a partir dos 03 anos de idade & 80 \\
\hline
\end{tabular}

A ESB e a coordenação definiram dias e períodos para a participação em reuniões, atendimento de escolares, adultos, visitas domiciliares. Havia extensão da triagem de risco de cárie dentária para escolares e grupos da unidade básica de saúde. Dentre as atividades educativas realizadas na comunidade, observadas in loco, estava a integração da Saúde Bucal com a Educação por meio do Programa de Saúde na Escola (PSE) com a escovação supervisionada realizada pelos ACS no ambiente escolar. As visitas domiciliares eram realizadas para acamados e indivíduos com dificuldade de locomoção. Diariamente, os cirurgiões-dentistas realizavam 0 lançamento da produção em programa de computador da UBS onde trabalhavam. A ESB participava semanalmente das reuniões de planejamento com os demais profissionais da unidade e mensalmente com a coordenação da saúde bucal para avaliação e acompanhamento dos resultados, por meio da análise dos relatórios de produção, indicadores e ações priorizadas.

Importante ressaltar que o incentivo financeiro recebido do PMAQ (Programa de Monitoramento e Avaliação da Qualidade) era repassado aos profissionais da Atenção Básica e que os cirurgiões-dentistas trabalhavam exclusivamente na ESF. Essas afirmações foram relatadas pelo gestor municipal e comparadas com as respostas das equipes de saúde bucal.

\section{AVALIAÇÃO DA AGENDA EM UMA UBS}

A agenda foi implantada no município em fevereiro de 2015 e avaliada quatro meses anteriores e quatro meses posteriores à sua implantação, período considerado para a prestação de contas quadrimestral em audiência pública. Na comparação entre os dois momentos foram obtidos os seguintes resultados: em relação aos pacientes atendidos na primeira consulta odontológica programática, verificou-se que houve aumento na cobertura em $63 \%$, redução de $30 \%$ dos casos de urgência e aumento de $11 \%$ nos tratamentos concluídos (Quadro 4). 


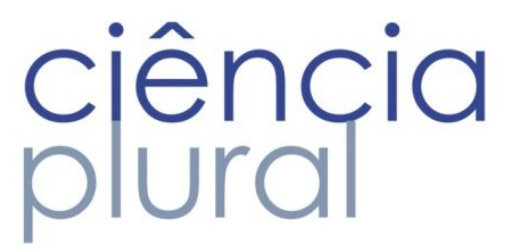

Quadro 4: Análise comparativa da produção da assistência odontológica prestada por uma ESB quatro meses antes e quatro meses após a implantação da agenda de saúde bucal

\begin{tabular}{|l|c|c|}
\hline Procedimentos & ESB 1: Novembro 2014 & ESB1: Junho 2015 \\
\hline Primeira consulta & $\mathrm{n}(\%)$ & $\mathrm{n}(\%)$ \\
\hline Consultas de urgência & $27(8,66)$ & $19(4,79)$ \\
\hline Tratamentos completados & $27(7,09)$ & $30(7,56)$ \\
\hline Total de procedimentos & $381(100 \%)$ & $397(100 \%)$ \\
\hline
\end{tabular}

\section{Discussão}

O estudo proporcionou a avaliação da efetividade de uma agenda de atividades de saúde bucal para a reorganização do processo de trabalho. Visa contribuir para a elaboração de políticas de atenção à saúde bucal, de forma a proporcionar um direcionamento das ações que não fiquem restritas apenas às quatro paredes do consultório odontológico.

Fatores positivos puderam ser ressaltados nesse estudo, como o trabalho em equipe, o protagonismo dos integrantes e da comunidade na construção das atividades e a ampliação do olhar crítico sobre a realidade social. ${ }^{8}$

A necessidade de implantação da agenda, ratificada na análise documental do Plano Municipal de Saúde Biênio 2014-2017 e no relatório do PMAQ-2013 (Programa de Monitoramento e Avaliação da Qualidade) realizado pelo Ministério da Saúde, demostra a preocupação do nível municipal e federal quanto a organização do processo de trabalho.

Observou-se que a apropriação do território de abrangência, das barreiras geográficas, do perfil populacional e da cobertura da equipe foram fundamentais para o planejamento e execução de ações com equidade. Conhecer o território implica em um processo de reconhecimento do espaço local e das relações da população da área de abrangência com a unidade de saúde ${ }^{9}$. Essa apropriação pode ser realizada por meio do Sistema de Informação da Atenção Básica (SIAB), que está sendo substituído pelo Sistema de Informação em Saúde para a Atenção Básica (SISAB) ${ }^{10}$, que contém informações sobre cadastros de famílias, condições de moradia e saneamento, situação de saúde, produção e composição das equipes de saúde. ${ }^{11}$

O território como locus da atenção é um desafio, principalmente pelo fato da população acreditar que o trabalho da equipe de saúde bucal é restrito ao atendimento no consultório odontológico. Como a implantação da saúde bucal na Estratégia da Saúde da Família ocorreu de modo tardio, há uma dificuldade da população em assimilar a Odontologia como integrante da equipe. É preciso que haja o envolvimento da equipe com a comunidade, informando-a e orientando-a quanto ao ingresso no sistema ${ }^{3}$ e sobre 0 tipo de modelo assistencial adotado pelo município, pois, quando as necessidades individuais não são resolvidas imediatamente, pode haver resistência e oposição dos usuários que não entendem claramente 0 objetivo do programa ${ }^{12}$. Inclusive, a percepção do usuário é de extrema importância ao se dimensionar o reflexo das ações que vêm sendo desenvolvidas no setor de saúde e serve como vetor de direcionamento e planejamento do serviço. ${ }^{13}$ 


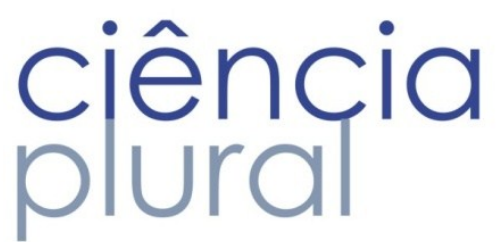

A agenda implantada contemplou atividades da equipe nos três eixos de atuação da ESF: unidade de saúde, comunidade e família. Dessa forma, possibilitou garantir que as ações de saúde bucal não sejam restritas ao equipamento odontológico, mas, incorporem outros atores sociais ${ }^{14}$.

A agenda da ESB foi discutida nos Conselhos Locais e Municipais de Saúde. Essa proximidade do usuário com os serviços e seus profissionais busca conhecer como o direito à saúde se expressa concretamente no cotidiano das pessoas. ${ }^{15}$

Durante oficina realizada para apresentação dos resultados e problematização da importância da agenda no planejamento e avaliação do processo de trabalho, os ACS demonstraram dúvidas se as atividades que exerciam estariam contempladas numa agenda de saúde bucal. As atividades educativas contempladas na agenda são realizadas na comunidade pelos ACS, sob a supervisão do cirurgião-dentista. Observou-se in loco a escovação supervisionada e a ação educativa realizada pelos ACS no ambiente escolar por meio da integração com o PSE: Programa de Saúde na Escola ${ }^{16}$. Para as atividades educativas de promoção e prevenção à saúde, recomenda-se o mínimo de dez participantes e duração mínima de 30 minutos ${ }^{17}$.

As visitas domiciliares estavam inseridas na agenda. A ampliação e qualificação das ações de saúde bucal também se fazem por meio de organização de visitas da equipe de saúde bucal às pessoas acamadas ou com dificuldades de locomoção, visando a identificação dos riscos e propiciando o acompanhamento e tratamento necessários 2 . A visita domiciliar não precisa acontecer em turnos pré-fixados, mas ser realizada sempre que necessário: gestante, acamado, recém-nascido, idoso, pessoas com necessidades especiais, previamente agendadas com 0 ACS. Cada localidade deve planejar a melhor estratégia para sua comunidade ${ }^{18}$, inclusive a lista de casos prioritários para a realização de visita domiciliar é solicitada na avaliação externa do PMAQ. ${ }^{6}$

As equipes realizavam a racionalização do fluxo por meio da triagem de risco. São consideradas triagens de risco as realizadas com grupos constituídos na comunidade, em domicílios, nos exames para diagnóstico de câncer bucal efetuados durante a Campanha de Vacinação dos Idosos ou em outros eventos que reúnam um contingente populacional ${ }^{17}$. É necessário desenvolver ações que priorizem os grupos e os fatores de risco com a finalidade de prevenir 0 aparecimento ou a persistência de doenças e danos evitáveis ${ }^{1}$. A lista de escolares identificados com necessidade de atendimento em saúde bucal e o registro de atividades realizadas em creches e/ou escolas são solicitados na avaliação externa do PMAQ. ${ }^{6}$

Para nortear a construção da agenda foram utilizados parâmetros em todas as equipes para 0 planejamento, programação e priorização das ações de saúde a serem desenvolvidas. A fim de que sejam realizadas a ampliação e a qualificação da assistência, recomenda-se que $75 \%$ a $85 \%$ das horas contratadas sejam dedicadas à assistência e $15 \%$ a $25 \%$ a outras atividades como planejamento, capacitação e atividades coletivas ${ }^{2}$. Quatro horas semanais devem ser utilizadas para a realização de reunião com todos os membros da equipe em conjunto ou não com a coordenação. 0 objetivo dessa atividade é permitir a discussão dos casos, planejamento das ações, avaliações, resolução de conflitos e troca de conhecimentos. Não são consideradas as reuniões dedicadas à operacionalização do fechamento de dados para o SIAB ${ }^{19}$. O planejamento dos serviços é importante para conferir direcionalidade às ações, às tomadas de decisões, racionalização de procedimentos e rotinas. Os parâmetros de planejamento e programação são referenciais quantitativos indicativos, sem qualquer caráter impositivo ou obrigatório, visando à equidade de acesso, a integralidade e a harmonização progressiva dos perfis da oferta das ações e serviços de saúde. Os parâmetros como referenciais quantitativos foram aprovados na Portaria 1.631 e podem subsidiar o gestor nos três níveis do governo. ${ }^{20}$

A agenda proporcionou uma visibilidade das atividades da equipe com grupos prioritários: gestantes, bebês, escolares, paciente especial, com hipertensão, com diabetes, beneficiário do Programa Bolsa Família. A priorização desses grupos para avaliação não significa atendimento exclusivo em detrimento dos demais 


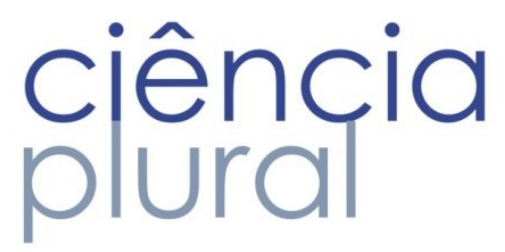

segmentos da população, promove apenas a organização da atenção, com vistas à universalização gradual do atendimento a toda a população da área de abrangência, com base no princípio da equidade. ${ }^{21} 0$ registro de acompanhamento de gestantes pela equipe de saúde bucal é solicitado na avaliação externa do PMAQ. ${ }^{6}$

Foi mantido 0 atendimento à demanda espontânea, o que possibilitou o apoio ao usuário que não está no universo priorizado para a Odontologia composto por área de risco social, escolas, creches, gestantes, grupos de desnutridos e outros grupos organizados 22 .

Quanto ao atendimento na atenção secundária o Centro de Especialidades Odontológicas (CEO) garantem as ações de média complexidade encaminhadas pelas equipes de saúde bucal.

$\mathrm{Na}$ análise comparativa da produção da assistência odontológica prestada quatro meses antes e doze meses após a implantação da agenda de saúde bucal em uma ESB, o aumento apresentado pelo indicador de primeira consulta odontológica programática foi importante, visto que, estima o acesso da população aos serviços odontológicos para assistência individual e pode subsidiar o gestor no planejamento, execução, implementação e avaliação das ações de saúde bucal. É relevante monitorar o número de procedimentos realizados, satisfação do usuário, demanda reprimida, discussão das demandas trazidas pelos ACS e pelos demais membros da equipe. A agenda precisa ser avaliada constantemente, pois poderá haver alterações das necessidades de acordo com 0 diagnóstico epidemiológico do território de atuação da equipe. A agenda é singular de cada equipe e poderá ser diferente por microáreas. Portanto, é importante que haja o preenchimento correto dos instrumentos de produção para um planejamento de acordo com a realidade local.

As diferenças verificadas na análise comparativa antes e após a implantação da agenda demonstraram que as consultas de urgência apresentaram redução, o que pode estar relacionado à inclusão de pacientes na rotina de atendimento preconizado na agenda de saúde bucal. Com a inserção da saúde bucal no processo de organização da atenção primária, a tendência é que haja uma diminuição gradual dos casos de urgência. ${ }^{21}$

Um aspecto positivo foi o acompanhamento do trabalho das equipes pelo coordenador de saúde bucal por meio dos relatórios de produção, indicadores e ações priorizadas com discussão dos resultados em reunião. Esse procedimento demonstra a responsabilidade do gestor para organizar o modelo de atenção à saúde bucal do município ao orientar os profissionais quanto às suas responsabilidades e cumprimento dos princípios e diretrizes do SUS na ESF ${ }^{3}$. Desse modo, o correto preenchimento do registro dos dados possibilita aos gestores e equipes de saúde o planejamento e avaliação das ações de saúde, garantindo a qualidade do serviço prestado. ${ }^{23}$

A gratificação do valor do incentivo financeiro do PMAQ era repassado aos funcionários das equipes de saúde da atenção básica com certificação ótimo e bom. Esse repasse financeiro promove a valorização profissional e a pontuação recebida no PMAQ ratifica a importância do trabalho em equipe na busca por resultados enriquecedores.

Embora a agenda tenha sido construída baseada na experiência local da ESF, poderá ser válida para outros modelos assistenciais, desde que sigam as diretrizes da Política Nacional da Atenção Básica e da Política Nacional da Saúde Bucal, com olhar crítico nos problemas locais, procurando soluções exequíveis, apoiadas na gestão e no controle social..$^{24}$

Esse estudo pode subsidiar o gestor que realiza a coordenação de saúde bucal municipal frente ao desafio de contemplar ações a serem executadas nos três eixos de atuação da ESF: UBS, família e comunidade e não restrito ao consultório odontológico, com o desenvolvimento de um trabalho equânime, universal e resolutivo. 


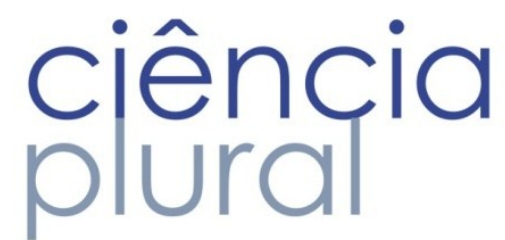

\section{Conclusão}

A agenda de saúde bucal apresentou resultados satisfatórios na reorganização do processo de trabalho para a melhoria do acesso aos serviços de saúde bucal. Resultou de um planejamento participativo, com as prioridades definidas de acordo com a situação epidemiológica e diagnóstico do território. Considerando que muitos gestores assumem o serviço sem conhecimento para o exercício da função, este estudo proporcionou uma orientação para o desenvolvimento de ações de saúde bucal, de acordo com as diretrizes e princípios do SUS. 


\section{ciência plural}

\section{Referências}

Brasil. Portaria $n^{0}$ 2. 488, de 21 de outubro de 2011. Aprova a política nacional de atenção básica, estabelecendo a revisão de diretrizes e normas para a organização da atenção básica, para a estratégia saúde da família (ESF) e o programa de agentes comunitários de saúde (PACS). Diário Oficial União 2011.

2. Brasil. Ministério da Saúde. Diretrizes da Política Nacional de Saúde Bucal. Brasilia: Ministério da Saúde; 2004. Disponível em: URL: http://bvsms.saude.gov.br/bvs/publicacoes/politica_nacional_brasil_sorridente.pdf

3. Pimentel FC, Albuquerque PC, Martelli PJL, Souza WV, Acioli RML. Caracterização do processo de trabalho das equipes de saúde bucal em municípios de Pernambuco, Brasil, segundo porte populacional: da articulação comunitária à organização do atendimento clínico. Cad Saúde Pública 2012; 28: 146- 57.

4. Brasil. Ministério da Saúde. Estratégia Saúde da Família- Equipe de Saúde Bucal. Brasilia: Ministério da Saúde; 2012. Disponivel em: URL:

http://dab.saude.gov.br/portaldab/ape_brasil_sorridente.php?conteudo=equipes

5. Ministério da Saúde (BR). Autoavaliação para Melhoria do Acesso e da Qualidade da Atenção Básica- AMAQ. Brasília (DF): Ministério da Saúde; 2012a. Disponível em: URL: http://189.28.128.100/dab/docs/geral/amaq.pdf

6. Ministério da Saúde (BR). Programa Nacional de Melhoria do Acesso e da Qualidade da Atenção Básica (PMAQ). Brasília (DF): Ministério da Saúde; 2012c. Disponível em: URL:

http://dab.saude.gov.br/portaldab/ape_pmaq.php

7. Bardin, L. Análise de conteúdo. Lisboa: Edições 70, 1979.

8. Barreto ICHC, Andrade LOM, Moreira AEMM, Machado MMT, Silva MRF, Oliveira RC et al. Gestão participativa no SUS e a integração ensino, serviço e comunidade: a experiência da Liga de Saúde da Família, Fortaleza, CE. Saúde Soc. 2012; 21: 80-93.

9. Brasil. Ministério da Saúde. Saúde bucal. Brasília: Ministério da Saúde; 2006. Disponível em: URL: http://189.28.128.100/dab/docs/publicacoes/cadernos_ab/abcad17.pdf

10. Brasil. Ministério da Saúde. SIAB- Sistema de Informação da Atenção Básica. Brasilia: Ministério da Saúde; 2008. Disponível em: URL: http://www2.datasus.gov.br/SIAB/index.php?area=01

11. Brasil. Portaria n $\mathrm{n}^{0} .412$, de 10 de julho de 2013. Institui o Sistema de Informação em Saúde para a Atenção Básica (SISAB). Diário Oficial União Brasília; 2013.

12. Narvai PC. Saúde Bucal: assistência ou atenção? Oficina do Grupo de Trabalho "Odontologia em Silos Sistemas Locais de Saúde". Rede CEDROS; 1992. Disponível em: URL:

http://www.ccs.ufsc.br/spb/os3_narvai.pdf 


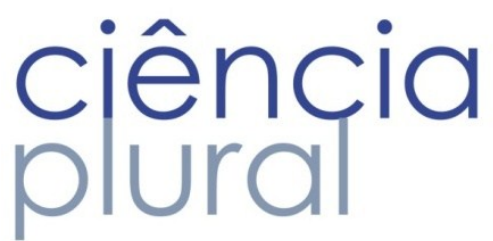

13. Moimaz SAS, Marques JAM, Saliba O, Garbin CAS, Zina LG, Saliba NA. Satisfação e percepção do usuário do SUS sobre o serviço público de saúde. Physis. 2010; 20: 1419-40.

14. Farfán J, Valenzuela MT. Incorporación de los odontólogos en los procesos de transformación al modelo de salud familiar de la atencion primaria en Chile. Rev Chil Salud Pública. 2014; 18: $72-80$.

15. Mattos GCM, Ferreira e Ferreira E, Leite ICG, Greco RM. A inclusão da equipe de saúde bucal na Estratégia Saúde da Família: entraves, avanços e desafios. Ciênc Saúde Coletiva. 2014; 19: 373-82.

16. Brasil. Decreto n ${ }^{0} 6.286$, de 5 de dezembro de 2007. Institui o Programa Saúde na Escola - PSE, e dá outras providências. Diário Oficial da União 2007.

17. São Paulo. Secretaria de Estado da Saúde de São Paulo. Manual de orientações em saúde bucal para o SUS no âmbito do estado de São Paulo. São Paulo: SES; 2010. Disponível em: URL:

http://www.saude.sp.gov.br/resources/ses/perfil/profissional-da-saude/grupo-tecnico-de-acoes-estrategicasgtae/saude-

bucal/documentostecnicos/manual_de_orientacoes_em_saude_bucal_para_o_sus_no_ambito_do_estado_de_ sao_paulo.pdf

18. Santos AM. Organização das ações em saúde bucal na estratégia de saúde da familia: ações individuais e coletivas baseadas em dispositivos relacionados e instituintes. Rev APS. 2006; 9: 190-200.

19. Brasil. Ministério da Saúde. Avaliação para melhoria da qualidade da estratégia saúde da família. Brasília: Ministério da Saúde; 2009.

20. Brasil. Portaria $n^{0} 1.631$, de $1^{\circ}$ de outubro de 2015. Aprova critérios e parâmetros para o planejamento e programação de ações e serviços de saúde no âmbito do SUS. Diário Oficial da União, 2015.

21. Minas Gerais. Secretaria de Estado da Saúde de Minas Gerais. Manual do Prontuário de saúde da família. Belo Horizonte (MG): SES/MG; 2007.

22. Roncalli AG. A organização da demanda em serviços públicos de saúde bucal: universalidade, equidade e integralidade em saúde bucal coletiva [tese]. Araçatuba (SP): Universidade Estadual Paulista "Júlio de Mesquita Filho"- Faculdade de Odontologia de Araçatuba; 2000.

23. Moimaz SAS, Ayach C, Garbin CAS, Saliba O. Auditoria na Saúde: justificativas de glosas no setor odontológico. J Health Sci Inst. 2012; 30: 112-6.

24. Medeiros LCMM. Educação permanente como instrumento de mudança na rede de atenção à saúde da família: um relato de experiência. Rev Ciência Plural 2015; 1(1): 65-74. 